\title{
Response to the Letter to the Editor by Dr. Srinivas on the recent publication of Al-Tawil et al. on the pharmacokinetics of transdermal buprenorphine patch in the elderly
}

\author{
Helen Johnson • Anna-Carin Berggren
}

Received: 28 February 2013 / Accepted: 18 March 2013 / Published online: 16 April 2013

(C) The Author(s) 2013. This article is published with open access at Springerlink.com

To the Editor,

We are writing in response to the letter from Dr. Srinivas commenting on the recent publication by AlTawil et al. on the pharmacokinetics of transdermal buprenorphine patch in the elderly [1]. We represent the authors and Mundipharma Research Ltd., the Clinical Pharmacology group of which, Helen Johnson (a coauthor) in particular, was responsible for the pharmacokinetic analysis carried out in this study.

The study reported comparable systemic exposures of buprenorphine and norbuprenorphine in two groups of elderly ( $\geq 75$ years) and younger (50-60 years) individuals, leading to the conclusion that no dosage alterations are necessary for pharmacokinetic (PK) reasons when treating elderly people with buprenorphine transdermal patches. We are surprised that Dr. Srinivas has elected to extrapolate from the average PK data that were reported in the article, based on the qualitatively (but not significantly) higher norbuprenorphine plasma concentrations in the elderly group, and subsequently to speculate that this may contribute to several safety issues, including respiratory depression.

In response, we wish to provide a more detailed illustration of the data generated in this study. The

\section{H. Johnson}

Clinical Pharmacology, Mundipharma Research Ltd.,

Cambridge Science Park, Milton Road,

Cambridge CB4 0GW England, UK

e-mail: helen.johnson@mundipharma-rd.eu

\section{A.-C. Berggren $(\bowtie)$}

Medical Affairs, Mundipharma AB, Mölndalsvägen 30B,

41263, Göteborg, Sweden

e-mail: anna-carin.berggren@mundipharma.se following figures illustrate the distribution of individual subject area under the curve values associated with buprenorphine and norbuprenorphine in the two study groups (Figs. 1 and 2).

These figures demonstrate very clearly that the ranges of exposure of both compounds were highly comparable between the groups, and illustrate why there were no significant differences recorded, or even hinted at, in the original analysis. The spread and overlap of individual Cmin and Cmax steady state data are also comparable between the two groups for both analytes, further reinforcing the primary conclusion. As stated in the original article, more adverse events (216) were actually recorded in the younger group than in elderly participants (164).

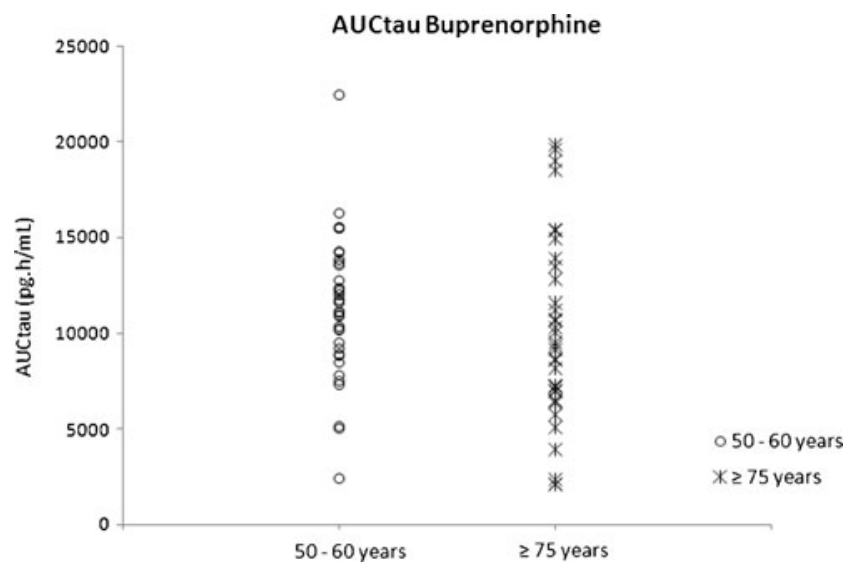

Fig. 1 Area under the plasma concentration-time curve at steady state (AUCtau) buprenorphine, data points are individual participant values 


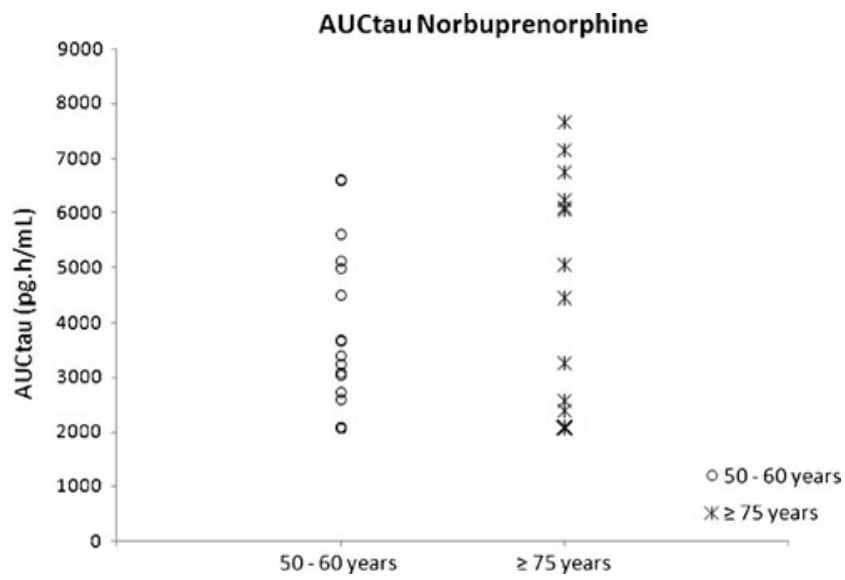

Fig. 2 Area under the plasma concentration-time curve at steady state (AUCtau) norbuprenorphine, data points are individual participant values

We have provided the data in more detail to support the original scientific findings. In so doing, we believe that we have demonstrated that there is no basis for the speculative text regarding norbuprenorphine and its safety profile in the elderly.

Yours sincerely,

Helen E Johnson (Clinical Pharmacokineticist) and Anna-Carin Berggren (Medical Affairs Manager).

Open Access This article is distributed under the terms of the Creative Commons Attribution License which permits any use, distribution, and reproduction in any medium, provided the original author(s) and the source are credited.

\section{References}

Al-Tawil N, Odar-Cederlöf I, Berggren A-C, Johnson HE (2013) Pharmacokinetics of transdermal buprenorphine patch in the elderly. Eur J Clin Pharmacol 69:143-149 